\title{
Lower Bounds of Periods of Periodic Solutions for a Class of Differential Equations with Variable Delays
}

\author{
Xin-Ge Liu and Mei-Lan Tang \\ School of Mathematics and Statistics, Central South University, Changsha 410083, China \\ Correspondence should be addressed to Mei-Lan Tang; csutmlang@163.com
}

Received 2 June 2013; Accepted 25 July 2013

Academic Editor: Nazim Idrisoglu Mahmudov

Copyright ( 2013 X.-G. Liu and M.-L. Tang. This is an open access article distributed under the Creative Commons Attribution License, which permits unrestricted use, distribution, and reproduction in any medium, provided the original work is properly cited.

Based on generalized Wirtinger's inequality, periods of periodic solutions of the nonautonomous differential equations with variable delays are investigated. Based on Hölder inequality, lower bounds of periods of periodic solutions for a class of functional differential equations with variable delays are obtained by a simple method.

\section{Introduction}

The existence and multiplicity of periodic solutions, bifurcations of periodic solutions, and stability of solutions of functional differential equations have attracted the attention of many mathematicians [1-5]. A lot of remarkable results have been achieved [6-10]. However, only a few works on periods of periodic solutions have been done (see, e.g., [11-13]). Suppose $f$ is Lipschitz continuous in a Banach space with constant $L$ and $x(t)$ is a $T$-periodic nonconstant solution of $x^{\prime}(t)=f(x(t))$. Lasota and Yorke [12] have showed that $T L \geq 4$. Busenberg et al. [14] refined the earlier estimate of $T L$ in [12]; they [14] showed that $T L \geq 6$. At the same time, they [14] also gave a simple proof of the better lower bound $T L \geq 2 \pi$ in spaces with the norm defined via an inner product. Mawhin and Walter [15] showed how some lower bounds on the period of the possible periodic solutions of autonomous ordinary differential equations due to Yorke [11] are easy consequences of the general principle. Zevin and Pinsky [16] investigated a class of Lipschitzian differential equations of even order; they obtained the minimal periods of periodic solutions. In 2012, Domoshnitsky et al. [17] investigated componentwise positivity of solutions to periodic boundary problem for linear functional differential system. Recently, Cheng and Zhang [18] proved a generalized Wirtingers inequality. Based on this inequality, they [18] studied estimates for lower bounds of periods of periodic solutions for the following autonomous delay differential equation:

$$
\dot{x}(t)=-\sum_{k=1}^{n} f(x(t-k r))
$$

where $x \in R^{p}, f \in C\left(R^{p}, R^{p}\right)$, and $r>0$ is a given constant. In their paper [18], delays are required to be constants with the form of $k r$. In this paper, we will replace the constant delay $k r$ with the generalized delay function $r_{k}(t)$ with $\left|r_{k}^{\prime}(t)\right|<1, k=1,2, \ldots, n$. Furthermore, the method used in our paper is simpler than that in [18]. Lower bounds of periods of periodic solutions for a class of functional differential equations with variable delays are obtained.

Consider the lower bounds of periods of periodic solutions for the following delay differential equations:

$$
\dot{x}(t)=-\sum_{k=1}^{n} f\left(x\left(t-r_{k}(t)\right)\right) \text {, }
$$

where $x \in R^{p}, f \in C\left(R^{p}, R^{p}\right), r_{k}(t)=r_{k}(t+T), r_{k}(t)>0$, and $\left|r_{k}^{\prime}(t)\right|<1$ for $t \in R$.

In order to estimate the lower bounds of periods of periodic solution of (2), we need the following definitions and lemmas. 
Definition 1. For a positive constant $L, f(x) \in C\left(R^{p}, R^{p}\right)$ is called $L$-Lipschitz continuous if, for all $x, y \in R^{p}$,

$$
|f(x)-f(y)| \leq L|x-y|,
$$

where $|\cdot|$ denotes the Euclidean norm in $R^{p}$.

Let $H_{T}^{1}\left(R, R^{p}\right)$ be the Hilbert space consisting of the $T$ periodic functions $x$ on $R$ which together with weak derivatives belong to $L^{2}\left(0, T ; R^{p}\right)$. For all $x, y \in L^{2}\left(0, T ; R^{p}\right)$, let $\langle x, y\rangle=\int_{0}^{T}(x, y) d t$ and $\|x\|=\sqrt{\langle x, x\rangle}$ denote the inner product and the norm in $L^{2}\left(0, T ; R^{p}\right)$, respectively, where $(\cdot, \cdot)$ is the inner product in $R^{p}$. Let $S=\{x \mid$ $\left.x(t)=\left(x_{1}(t), x_{2}(t), \ldots, x_{p}(t)\right)\right\}$, where $x_{i}(t)$ has the second derivative, $i=1,2, \ldots, p$.

Lemma 2 (see [8]). Suppose $\tau \in C_{\omega}^{1}$ and $\tau^{\prime}(t)<1$, for all $t \in[0, \omega]$. Then the function $t-\tau(t)$ has an inverse $\mu(t)$ satisfying $\mu \in C(R, R)$ with $\mu(a+\omega)=\mu(a)+\omega$.

Lemma 3 (see [18]). If $x \in H_{T}^{1}$ and $\int_{0}^{T} x(t) d t=0$, then

$$
\int_{0}^{T}|x(t)|^{2} d t \leq \frac{T^{2}}{4 \pi^{2}} \int_{0}^{T}|\dot{x}(t)|^{2} d t
$$

\section{Main Results}

Since $r_{k}^{\prime}(t)<1$, by Lemma 2 , the inverse of $t-r_{k}(t)$ exists. Let $\mu_{k}(s)$ be the inverse of $t-r_{k}(t)$.

Theorem 4. Let $x$ be a nonconstant T-periodic solution of the nonautonomous delay differential equation (2) and $x \in$ S. Suppose that the function $f: R^{p} \rightarrow R^{p}$ is L-Lipschitz continuous and $r_{k}(t)=r_{k}(t+T), r_{k}(t)>0,\left|r_{k}^{\prime}(t)\right|<1$. Then $T>\sqrt{2} \pi / n L$.

Proof. Since $x$ is a nonconstant $T$-periodic solution of the nonautonomous delay differential equation (2), for all $t, u \in$ $R$, we have

$$
\begin{aligned}
|\dot{x}(t+u)-\dot{x}(t)| & \\
& =\left|\sum_{k=1}^{n} f\left(x\left(t+u-r_{k}(t+u)\right)\right)-\sum_{k=1}^{n} f\left(x\left(t-r_{k}(t)\right)\right)\right| \\
& \leq \sum_{k=1}^{n}\left|f\left(x\left(t+u-r_{k}(t+u)\right)\right)-f\left(x\left(t-r_{k}(t)\right)\right)\right| \\
& \leq L \sum_{k=1}^{n}\left|x\left(t+u-r_{k}(t+u)\right)-x\left(t-r_{k}(t)\right)\right| .
\end{aligned}
$$

We claim that if $u \neq 0$, then, for $t \in R$, there exists at least one $k$ such that

$$
u-r_{k}(t+u)+r_{k}(t) \neq 0 .
$$

Otherwise, if $u-r_{k}(t+u)+r_{k}(t)=0$ for $k=1,2, \ldots, n$, then

$$
x\left(t+u-r_{k}(t+u)\right)-x\left(t-r_{k}(t)\right)=0 .
$$

From (5), one has

$$
|\dot{x}(t+u)-\dot{x}(t)|=0
$$

Noting that $x \in S$, we obtain

$$
|\ddot{x}(t)|=0 .
$$

Noting that $x(t)=x(t+T)$, then $\int_{0}^{T} \dot{x}(t) d t=0$. From Lemma 3, we have

$$
2 \pi\|\dot{x}\| \leq T\|\ddot{x}\| .
$$

Then $|\dot{x}|=0 . x$ is a constant $T$-periodic solution. This contradicts the assumption that $x$ is a nonconstant $T$-periodic solution.

For simplicity of proof, we suppose that $u-r_{k}(t+u)+$ $r_{k}(t) \neq 0$ for $k=1,2, \ldots, n,(5)$ can be rewritten as

$$
\begin{gathered}
\left|\frac{\dot{x}(t+u)-\dot{x}(t)}{u}\right| \\
\leq L \sum_{k=1}^{n}\left|\frac{x\left(t+u-r_{k}(t+u)\right)-x\left(t-r_{k}(t)\right)}{u-r_{k}(t+u)+r_{k}(t)}\right| \\
\times\left|\frac{u-r_{k}(t+u)+r_{k}(t)}{u}\right| .
\end{gathered}
$$

Let $u \rightarrow 0$; one has

$$
\begin{aligned}
|\ddot{x}(t)| & \leq L \sum_{k=1}^{n}\left|\dot{x}\left(t-r_{k}(t)\right)\right|\left[1-r_{k}^{\prime}(t)\right] \\
& =L \sum_{k=1}^{n}\left|\dot{x}\left(t-r_{k}(t)\right)\right|\left[1-r_{k}^{\prime}(t)\right]^{1 / 2}\left[1-r_{k}^{\prime}(t)\right]^{1 / 2} .
\end{aligned}
$$

Applying Hölder inequality gives

$$
\begin{aligned}
|\ddot{x}(t)| & \leq L \sum_{k=1}^{n}\left|\dot{x}\left(t-r_{k}(t)\right)\right|\left[1-r_{k}^{\prime}(t)\right]^{1 / 2}\left[1-r_{k}^{\prime}(t)\right]^{1 / 2} \\
\leq & L\left\{\sum_{k=1}^{n}\left|\dot{x}\left(t-r_{k}(t)\right)\right|^{2}\left[1-r_{k}^{\prime}(t)\right]\right\}^{1 / 2} \\
& \times\left\{\sum_{k=1}^{n}\left[1-r_{k}^{\prime}(t)\right]\right\}^{1 / 2} \\
< & (2 n)^{1 / 2} L\left\{\sum_{k=1}^{n}\left|\dot{x}\left(t-r_{k}(t)\right)\right|^{2}\left[1-r_{k}^{\prime}(t)\right]\right\}^{1 / 2} .
\end{aligned}
$$

Raising both sides of inequality (13) to power 2 and integrating both sides from 0 to $T$, we have

$$
\begin{aligned}
& \int_{0}^{T}|\ddot{x}(t)|^{2} d t \\
& \quad<2 n L^{2} \int_{0}^{T} \sum_{k=1}^{n}\left|\dot{x}\left(t-r_{k}(t)\right)\right|^{2}\left[1-r_{k}^{\prime}(t)\right] d t .
\end{aligned}
$$


Since $\mu_{k}(s)$ is the inverse of $t-r_{k}(t)$, by using Lemma 2 , we have

$$
\begin{aligned}
\int_{0}^{T}|\ddot{x}(t)|^{2} d t & \\
& <2 n L^{2} \sum_{k=1}^{n} \int_{0}^{T}\left|\dot{x}\left(t-r_{k}(t)\right)\right|^{2}\left[1-r_{k}^{\prime}(t)\right] d t \\
& =2 n L^{2} \sum_{k=1}^{n} \int_{-r_{k}(0)}^{T-r_{k}(T)}|\dot{x}(s)|^{2} \\
& =2 n L^{2} \sum_{k=1}^{n} \int_{-r_{k}(0)}^{T-r_{k}(T)}|\dot{x}(s)|^{2} d s \\
& =2 n L^{2} \sum_{k=1}^{n} \int_{0}^{T}|\dot{x}(s)|^{2} d s \\
& =2 n^{2} L^{2} \int_{0}^{T}|\dot{x}(s)|^{2} d s .
\end{aligned}
$$

That is,

$$
\|\ddot{x}\|<\sqrt{2} n L\|\dot{x}\| .
$$

Since $x(t)=x(t+T)$, obviously, $\int_{0}^{T} \dot{x}(t) d t=0$. By Lemma 3, we have $2 \pi\|\dot{x}\| \leq T\|\ddot{x}\|$. So

$$
T>\frac{\sqrt{2} \pi}{n L}
$$

Remark 5. When delay $r_{k}(t)=k r, k=1,2, \ldots, n$, from the second inequality of (13), we can easily obtain Theorem 1 in [18].

We can easily obtain the following result.

Corollary 6. Let $x$ be a nonconstant T-periodic solution of the nonautonomous delay differential equation (2) and $x \in$ S. Suppose that the function $f: R^{p} \rightarrow R^{p}$ is L-Lipschitz continuous and $r_{k}(t)=r_{k}(t+T), r_{k}(t)>0,-1 / n \leq r_{k}^{\prime}(t)<1$. Then $T \geq 2 \pi / \sqrt{n(n+1)} L$.

\section{Acknowledgments}

The authors are grateful to the referees for their valuable comments. This study was partly supported by NSFC under Grant nos. 61271355 and 61070190, the ZNDXQYYJJH under Grant no. 2010QZZD015, and NFSS under Grant no. 10BJL020.

\section{References}

[1] M. Han, "Bifurcations of periodic solutions of delay differential equations," Journal of Differential Equations, vol. 189, no. 2, pp. 396-411, 2003.
[2] K. Gopalsamy, X. Z. He, and L. Z. Wen, "On a periodic neutral logistic equation," Glasgow Mathematical Journal, vol. 33, no. 3, pp. 281-286, 1991.

[3] P. Dormayer, "The stability of special symmetric solutions of $\dot{x}(t)=\alpha f(x(t-1))$ with small amplitudes," Nonlinear Analysis. Theory, Methods \& Applications, vol. 14, no. 8, pp. 701-715, 1990.

[4] J. Li, X.-Z. He, and Z. Liu, "Hamiltonian symmetric groups and multiple periodic solutions of differential delay equations," Nonlinear Analysis. Theory, Methods \& Applications, vol. 35, no. 4, pp. 457-474, 1999.

[5] G. Fei, "Multiple periodic solutions of differential delay equations via Hamiltonian systems. I," Nonlinear Analysis. Theory, Methods \& Applications, vol. 65, no. 1, pp. 25-39, 2006.

[6] Y. Kuang and A. Feldstein, "Boundedness of solutions of a nonlinear nonautonomous neutral delay equation," Journal of Mathematical Analysis and Applications, vol. 156, no. 1, pp. 293304, 1991.

[7] Z. Liu and Y. Mao, "Existence theorem for periodic solutions of higher order nonlinear differential equations," Journal of Mathematical Analysis and Applications, vol. 216, no. 2, pp. 481490, 1997.

[8] S. Lu and W. Ge, "Existence of positive periodic solutions for neutral logarithmic population model with multiple delays," Journal of Computational and Applied Mathematics, vol. 166, no. 2, pp. 371-383, 2004.

[9] Y. Luo and Z. Luo, "Existence of positive periodic solutions for neutral multi-delay logarithmic population model," Applied Mathematics and Computation, vol. 216, no. 4, pp. 1310-1315, 2010.

[10] M.-L. Tang, X.-G. Liu, and X.-B. Liu, "New results on periodic solutions for a kind of Rayleigh equation," Applications of Mathematics, vol. 54, no. 1, pp. 79-85, 2009.

[11] J. A. Yorke, "Periods of periodic solutions and the Lipschitz constant," Proceedings of the American Mathematical Society, vol. 22, pp. 509-512, 1969.

[12] A. Lasota and J. A. Yorke, "Bounds for periodic solutions of differential equations in Banach spaces," Journal of Differential Equations, vol. 10, pp. 83-91, 1971.

[13] T. Y. Li, "Bounds for the periods of periodic solutions of differential delay equations," Journal of Mathematical Analysis and Applications, vol. 49, pp. 124-129, 1975.

[14] S. N. Busenberg, D. C. Fisher, and M. Martelli, "Better bounds for periodic solutions of differential equations in Banach spaces," Proceedings of the American Mathematical Society, vol. 98, no. 2, pp. 376-378, 1986.

[15] J. Mawhin and W. Walter, "A general symmetry principle and some implications," Journal of Mathematical Analysis and Applications, vol. 186, no. 3, pp. 778-798, 1994.

[16] A. A. Zevin and M. A. Pinsky, "Minimal periods of periodic solutions of some Lipschitzian differential equations," Applied Mathematics Letters, vol. 22, no. 10, pp. 1562-1566, 2009.

[17] A. Domoshnitsky, R. Hakl, and J. Sremr, "Component-wise positivity of solutions to periodic boundary value problem for linear functional differential systems," Journal of Inequalities and Applications, vol. 2012, article 112, 2012.

[18] R. Cheng and D. Zhang, "A generalized Wirtinger's inequality with applications to a class of ordinary differential equations," Journal of Inequalities and Applications, vol. 2009, Article ID 710475, 7 pages, 2009. 


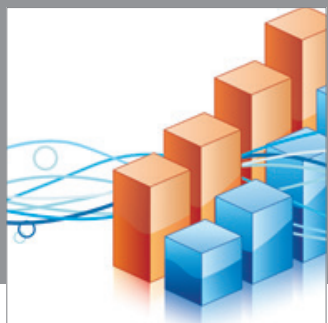

Advances in

Operations Research

mansans

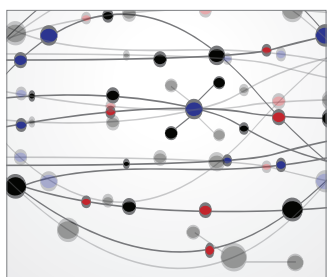

The Scientific World Journal
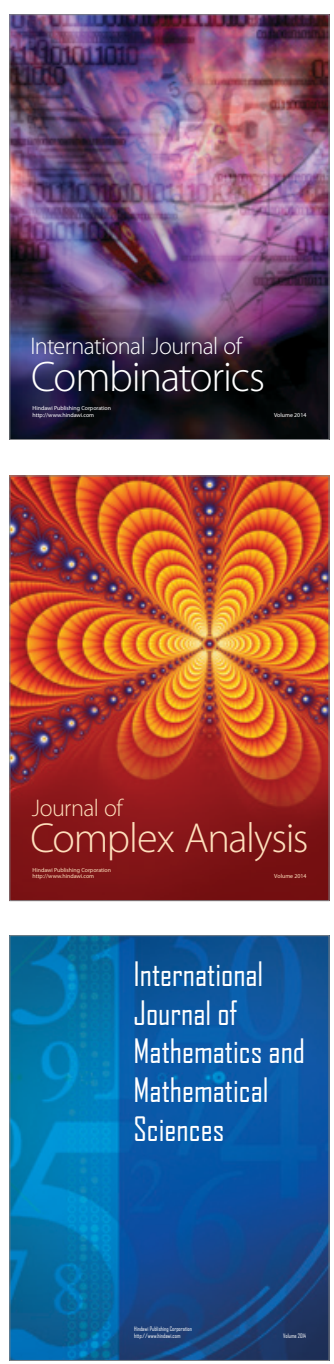
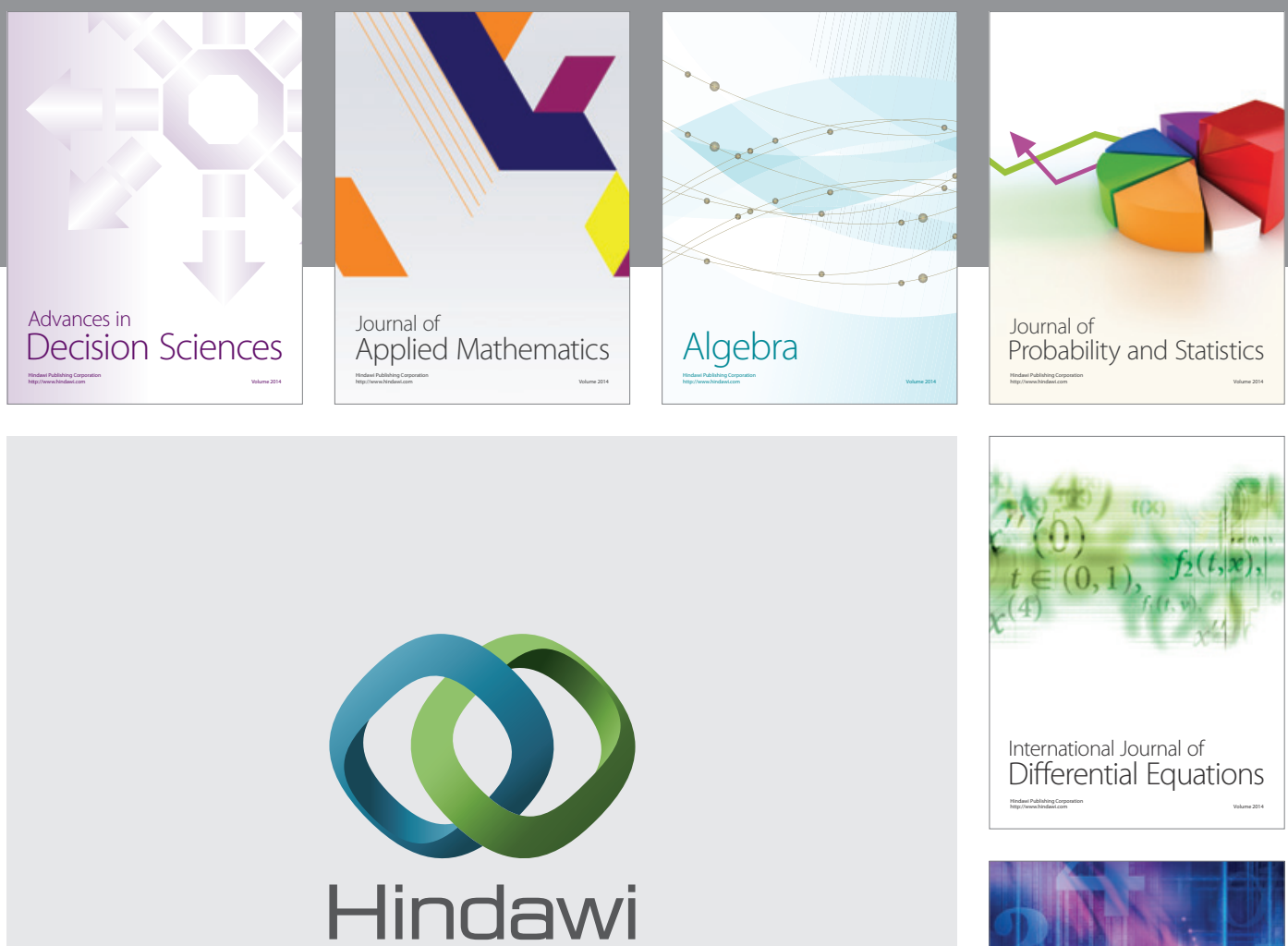

Submit your manuscripts at http://www.hindawi.com
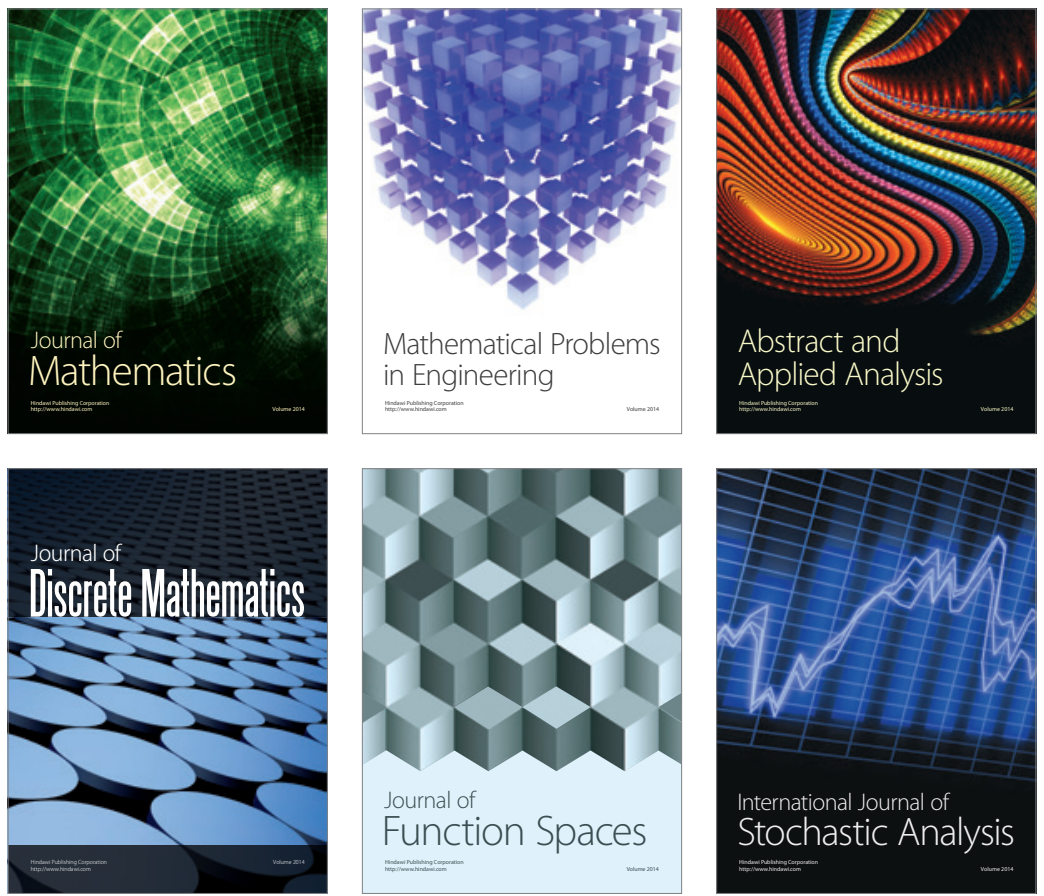

Journal of

Function Spaces

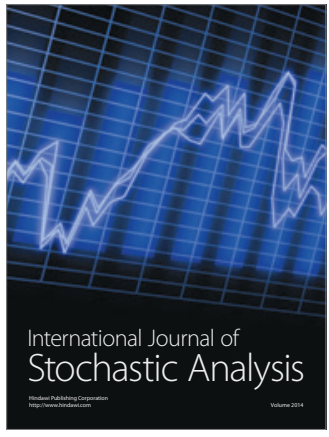

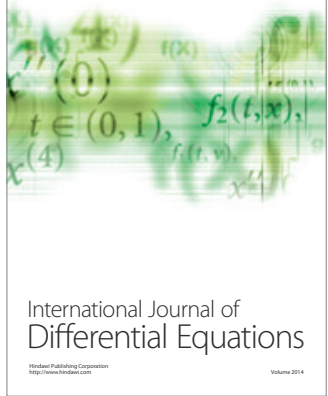
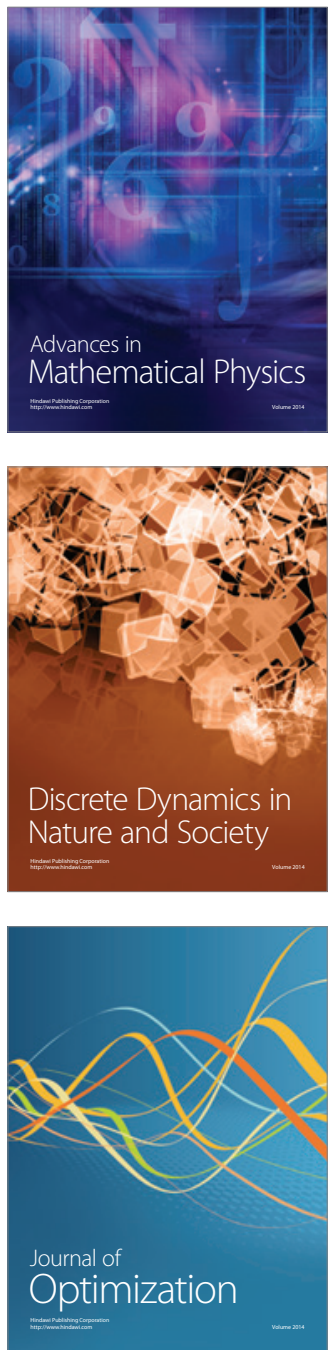